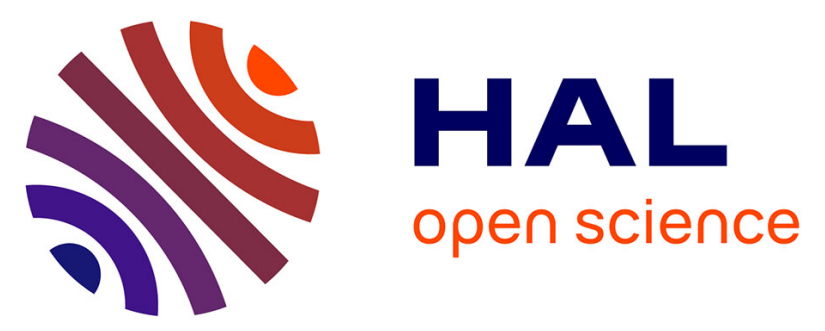

\title{
Molecular dynamics study of rare-earth doped Mg-silicate nanoparticles in vitreous silica: from the preform to the fiber
}

Jérémy Turlier, Stéphane Chaussedent, Xavier Bidault, Natalie Gaumer, Dominique Guichaoua, Patrice Raso, Hussein Fneich, Ahmad Mehdi, Daniel Neuville, Manuel Vermillac, et al.

\section{To cite this version:}

Jérémy Turlier, Stéphane Chaussedent, Xavier Bidault, Natalie Gaumer, Dominique Guichaoua, et al. Molecular dynamics study of rare-earth doped Mg-silicate nanoparticles in vitreous silica: from the preform to the fiber. Fiber Lasers and Glass Photonics: Materials through Applications, Apr 2018, Strasbourg, France. 10.1117/12.2306777 . hal-01857971

\section{HAL Id: hal-01857971 \\ https://hal.science/hal-01857971}

Submitted on 17 Aug 2018

HAL is a multi-disciplinary open access archive for the deposit and dissemination of scientific research documents, whether they are published or not. The documents may come from teaching and research institutions in France or abroad, or from public or private research centers.
L'archive ouverte pluridisciplinaire HAL, est destinée au dépôt et à la diffusion de documents scientifiques de niveau recherche, publiés ou non, émanant des établissements d'enseignement et de recherche français ou étrangers, des laboratoires publics ou privés. 


\title{
Molecular Dynamics study of rare-earth doped Mg-silicate nanoparticles in vitreous silica: from the preform to the fiber
}

\author{
J. Turlier ${ }^{\mathrm{a}}$, S. Chaussedent ${ }^{\mathrm{a}^{*}}$, X. Bidault ${ }^{\mathrm{a}}$, N. Balu Gaumer ${ }^{\mathrm{a}}$, D. Guichaoua ${ }^{\mathrm{a}}$, P. Raso ${ }^{\mathrm{a}}, \mathrm{H}$. \\ Fneich $^{\mathrm{b}}$, A. Mehdi ${ }^{\mathrm{b}}$, D.R. Neuville ${ }^{\mathrm{c}}$, M. Vermillac ${ }^{\mathrm{d}}$, and W. Blanc ${ }^{\mathrm{d}}$ \\ a'Université d'Angers, Laboratoire de Photonique d'Angers (LPhiA)-UPRES EA 4464, 2 Bd \\ Lavoisier, 49045 Angers Cedex 01, France

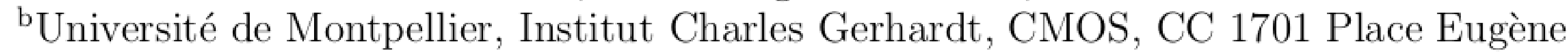 \\ Bataillon, 34095 Montpellier Cedex 5, France \\ ${ }^{c}$ Géomatériaux, CNRS-IPGP, Sorbonne Paris-Cité, 1 Rue Jussieu, 75005 Paris, France \\ dUniversité Côte d'Azur, INPHYNI-CNRS UMR 7010, Parc Valrose, 06108 Nice, France
}

\begin{abstract}
Formation of rare-earth doped nanoparticles into silica matrix has been modelized by Molecular Dynamics simulations. Preforms with molar composition $0.10 \mathrm{MgO}-0.90 \mathrm{SiO}_{2}$ and $0.01 \mathrm{EuO}_{3 / 2}-0.10 \mathrm{MgO}-0.89 \mathrm{SiO}_{2}$ have been investigated to have an insight on the structure and chemical composition of the nanoparticles, as well as the rare-earth ions local environment and their clustering. We have finally applied a uniaxal elongation of the rare-earth doped preform in order to mimic the drawing step that changes a preform into a fiber. We present herein first results on the modification of the nanoparticles size distribution.
\end{abstract}

Keywords: Molecular Dynamics, rare-earth doped glass, silicate nanoparticles, silica deformation

\section{INTRODUCTION}

Rare-earth (RE) doped glasses are of particular interest for optical components such as optical amplifiers and fiber lasers. Nowadays technological challenges (massification of telecommunication network for instance) require material having "augmented" intrinsic optical properties while keeping low manufacturing cost. To address these issues, a new family of optical fiber lasers has been developed for few years, consisting in incorporating RE-doped nanoparticles (NP) in silica glassy matrix. On one hand, the material takes advantage of the mechanical, chemical and optical properties of silica at low cost. On the other hand, NPs can allow an engineering of the spectroscopic response through a control of the NP composition and structure.

A flexible technique to manufacture these fibers consists to embed rare-earth ions within oxide NPs, which grow in-situ within silica-based preform prepared by MCVD (modified chemical vapor deposition). ${ }^{1}$ This growing process relies on phase separation phenomenon in silicate system containing alkaline earth elements $(\mathrm{Mg}, \mathrm{Ca}$, $\mathrm{Sr})$. Using this approach, Blanc et al. ${ }^{2}$ report on modifications of the $\mathrm{Er}^{3+}$ ions spectroscopy in $\mathrm{Mg}$-silicate NPs doped into optical fiber preforms. These modifications are related to the different local environments experienced by luminescent ions. Furthermore, incorporation of network modifier elements into silica glass limits the formation of rare-earth clusters, ${ }^{3}$ known to lead to a quenching of the luminescence intensity by means of ion-ion interaction mechanisms.

Nanoparticles are already present in the preform and modifications of their shape during the drawing process have been observed. ${ }^{4}$ Rheological and thermodynamical effects of the drawing step on the morphological properties of the NPs may lead to a new strategy to control the size distribution of the NPs in the fiber.

In this work, we used Molecular Dynamics (MD) simulations to have insight into the local environment of RE ions and into the structure of $\mathrm{Mg}$-silicate nanoparticles within the preform as well as within the fiber. For this purpose, we used a simple and adaptive potential ${ }^{5}$ that allowed us to reproduce the phase separation of the $x \mathrm{MgO}-(1-$ $x) \mathrm{SiO}_{2}$ binary glass. ${ }^{6}$ Through melt/quench protocol, We elaborated two preforms with different chemical compositions: $0.10 \mathrm{MgO}-0.90 \mathrm{Si}_{2}$ and $0.01 \mathrm{EuO}_{3 / 2}-0.10 \mathrm{MgO}-0.89 \mathrm{SiO}_{2}$. Then we applied on the europium doped preform an uniaxial elongation at different temperatures in order to give an insight of the drawing effect on the nanoparticles.

\footnotetext{
* stephane.chaussedent@univ-angers.fr
} 


\section{MD SIMULATIONS}

\subsection{Interatomic potential and simulation techniques}

Classical Molecular Dynamics simulations were carried out by the LAMMPS ${ }^{7}$ (Large-scale Atomic/Molecular Massively Parallel Simulator) package, with periodic boundary conditions. Velocity-Störmer-Verlet algorithm with a time step of 1.0 fs was used to integrate Newton's equations of motion. All simulations were performed using a modified version of the interatomic potential developed by Pedone $\mathrm{et} \mathrm{al.},{ }^{8}$ designed to reproduce structural and mechanical properties of silicates. ${ }^{9}$ The original version was given by a sum of a long-range Coulomb term, a short-range Morse function, and a repulsive contribution. Thus the potential between two particles was given by

$$
U(r)=\frac{q_{i} q_{j}}{r}+D_{i j}\left[\left\{1-e^{-a_{i j}\left(r-r_{0}\right)}\right\}^{2}-1\right]+\frac{C_{i j}}{r^{12}},
$$

where $i$ and $j$ are atom types, $q_{i}$ and $q_{j}$ are partial charges, $D_{i j}, C_{i j}, a_{i j}$ and $r_{0}$ are adjustable parameters and $r$ is the interatomic distance. Short range interactions were truncated at $5.5 \AA$. The Coulomb part was evaluated using the Wolf method ${ }^{10}$ with a long-range cutoff set as $7.5 \AA$ and a damping parameter set as 0.30 $\AA^{-1}$. In order to reproduce the phase separation phenomenon in the $x \mathrm{MgO}-(1-x) \mathrm{SiO}_{2}$ binary glasses, we used the modified version of the Pedone potential as proposed by Bidault et al.: $:^{5}$ this model takes into account the different ionicity of $\mathrm{Si}-\mathrm{O}$ and $\mathrm{Mg}-\mathrm{O}$ bonds, which is known to be crucial to model the phase separation phenomenon. ${ }^{11}$ Independently of the $\mathrm{MgO}$ concentration, a unique set of potential parameters is defined, and variations of the bond ionicity are modeled in adapting regularly each oxygen partial charge as a function of its surrounded cationic environment. Details on the procedure can be found in Ref. 5 .

\subsection{Preform preparation}

Preform preparation was done at constant pressure and temperature using Nosé-Hoover barostat and thermostat. Silica glass was obtained by melting and equilibrating a crystalline silica structure at $4000 \mathrm{~K}$ during $0.1 \mathrm{~ns}$. From $4000 \mathrm{~K}$, the melt was continuously cooled down at $5 \mathrm{~K} \mathrm{ps}^{-1}$ and finally equilibrated at $300 \mathrm{~K}$. Oxygen, magnesium and rare-earth atoms were placed randomly in this silica matrix to model the desired chemical composition. Finally, another melt/quench sequence was performed in order to allow the phase separation: the system was melted at $4000 \mathrm{~K}$ during $0.1 \mathrm{~ns}$ and quenched at $0.5 \mathrm{~K} \cdot \mathrm{ps}^{-1}$ from $4000 \mathrm{~K}$ to $2400 \mathrm{~K}$. Then, long stages of $6.4 \mathrm{~ns}$ are performed at $2400 \mathrm{~K}, 2300 \mathrm{~K}, 2200 \mathrm{~K}, 2100 \mathrm{~K}, 2000 \mathrm{~K}, 1900 \mathrm{~K}$, which is a temperature range wherein the phase separation occurs. Finally the system is quenched at $0.5 \mathrm{~K} / \mathrm{ps}$ from $1900 \mathrm{~K}$ to $300 \mathrm{~K}$ to get the preform structure. Using the same numerical protocol, preforms with molar composition $0.10 \mathrm{MgO}-0.90 \mathrm{Si}_{2}(2916 \mathrm{Mg})$ and $0.01 \mathrm{Eu}_{3 / 2^{-}}$ $0.10 \mathrm{MgO}-0.89 \mathrm{SiO}_{2}(2952 \mathrm{Mg}$ and $306 \mathrm{Eu})$ were obtained. The final volume at $\mathrm{T}=300 \mathrm{~K}$ is approximately $150 \times 50 \times 150 \AA^{3}$ (Fig.1).

\subsection{Uniaxal elongation}

We applied on the rare-earth doped preform a numerical protocol to mimic the experimental drawing process of optical fibers. ${ }^{12}$ The preform was heated and equilibrated during $0.1 \mathrm{~ns}$ at three different drawing temperatures around the calculated glass transition temperature $\mathrm{T}_{\mathrm{g}}=2120 \mathrm{~K}$, i.e. $\mathrm{T}=1900 \mathrm{~K}, \mathrm{~T}=2150 \mathrm{~K}$ and $\mathrm{T}=2300 \mathrm{~K}$. We did not exceed $2300 \mathrm{~K}$ (even if the calculated softening temperature of our glass model is $\mathrm{T}_{\mathrm{s}}=2945 \mathrm{~K}$ ) in order to preserve the main structural features of the preform. Then, a uniaxal elongation was applied by means of an anisotropic NPT algorithm reproducing a natural deformation along the $y$-axis: in this direction the box length evolved as $L_{y}(t)=L_{0 y} e^{\nu t}$, with a strain rate $\nu=10^{9} \mathrm{~s}^{-1}$, while no stress was imposed to lateral sides. The strain $\epsilon=\mathrm{e}^{\nu \mathrm{t}}-1$ was 8 at the end of the elongation and then the fiber was cooled down to $300 \mathrm{~K}$ at 1 K.ps ${ }^{-1}$. Final fiber dimensions were 50 x 450 x $50 \AA^{3}$.

\section{RESULTS}

The global structure of the $0.10 \mathrm{MgO}-0.90 \mathrm{Si}_{2}$ binary glass is shown in Fig. 1 . We can see the existence of two different kinds of region ; on one hand, Mg-rich Si-poor phases ; on the other hand, Si-rich Mg-poor phases, in agreement with experimental observations. ${ }^{14,15}$ In this system, $63.6 \%$ of Si atoms are located at a distance longer than $4.0 \AA$ (first minimum of $\mathrm{Mg}$-Si radial distribution fonction (RDF)) from $\mathrm{Mg}$ atoms. In the case of randomly distributed $\mathrm{Mg}$ atoms within a silica matrix (i.e., without phase separation), only $42.0 \%$ of $\mathrm{Si}$ atoms are "demixed". Thus, the phase separation is evidenced by the difference between these two percentages. 

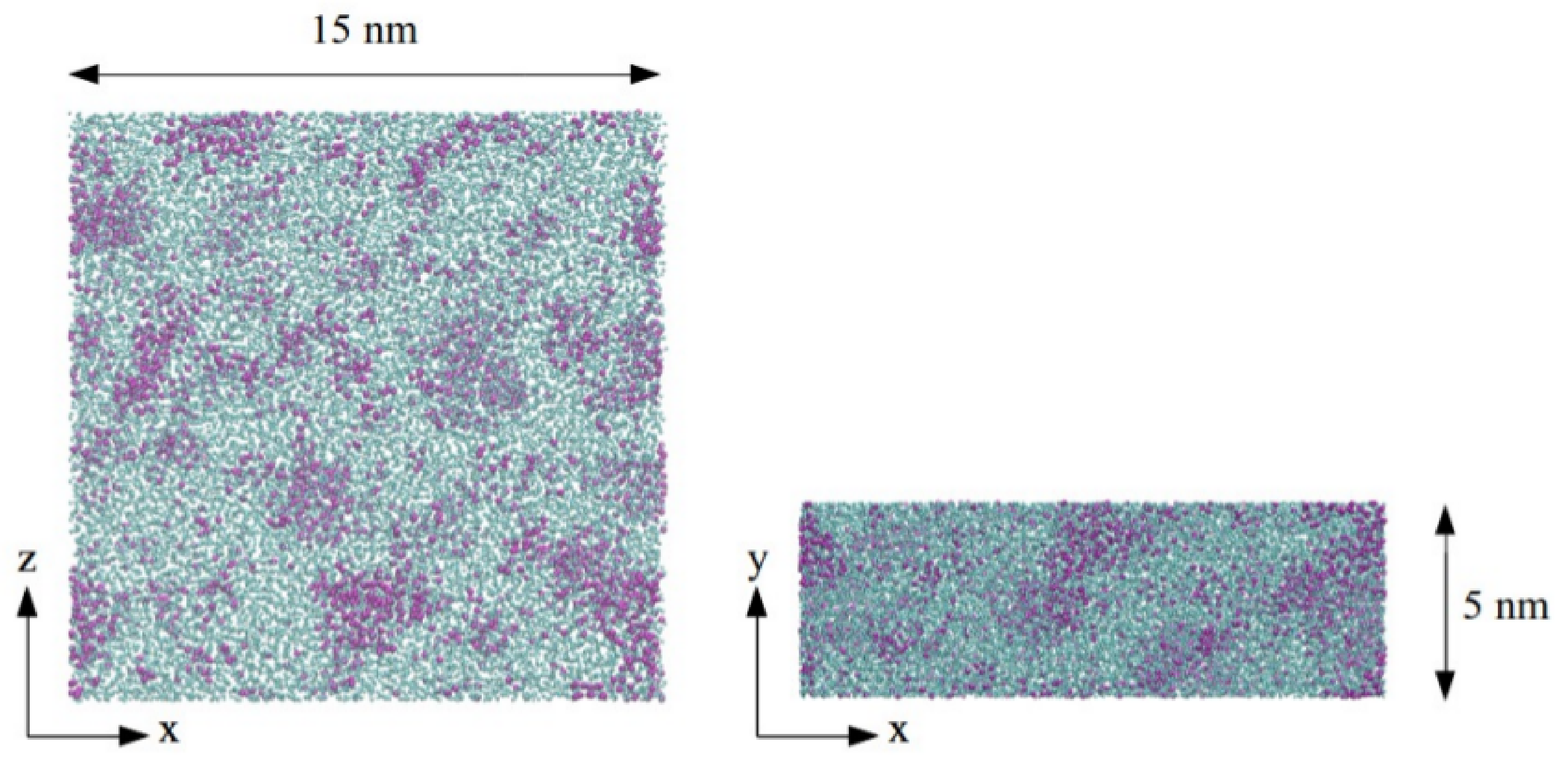

Figure 1. Image of $0.10 \mathrm{MgO}-0.90 \mathrm{Si}_{2}$ glass model. Purple spheres show $\mathrm{Mg}$ atoms while cyan spheres represent Si atoms. $\mathrm{O}$ atoms are not shown for the sake of clarity. These images were created using VMD software. ${ }^{13}$

\subsection{Nanoparticles structures and compositions}

The previously identified Mg-rich phases are inhomogeneously distributed in a Mg-poor silica matrix. In such a way, these regions can be considered as nanoparticles (NP). As shown in Fig. 2, they are amorphous, with non spherical shapes, and exhibit a wide range of sizes. The biggest one is composed of $343 \mathrm{Mg}$. About $10 \%$ of $\mathrm{Mg}$ atoms are isolated into a pure silica phase and about $50 \%$ are involved into NP composed of $126 \mathrm{Mg}$ atoms and more.

To investigate the $\mathrm{Mg}^{2+}$ ion local environment, we have defined different kinds of oxygen species (Fig. 3).

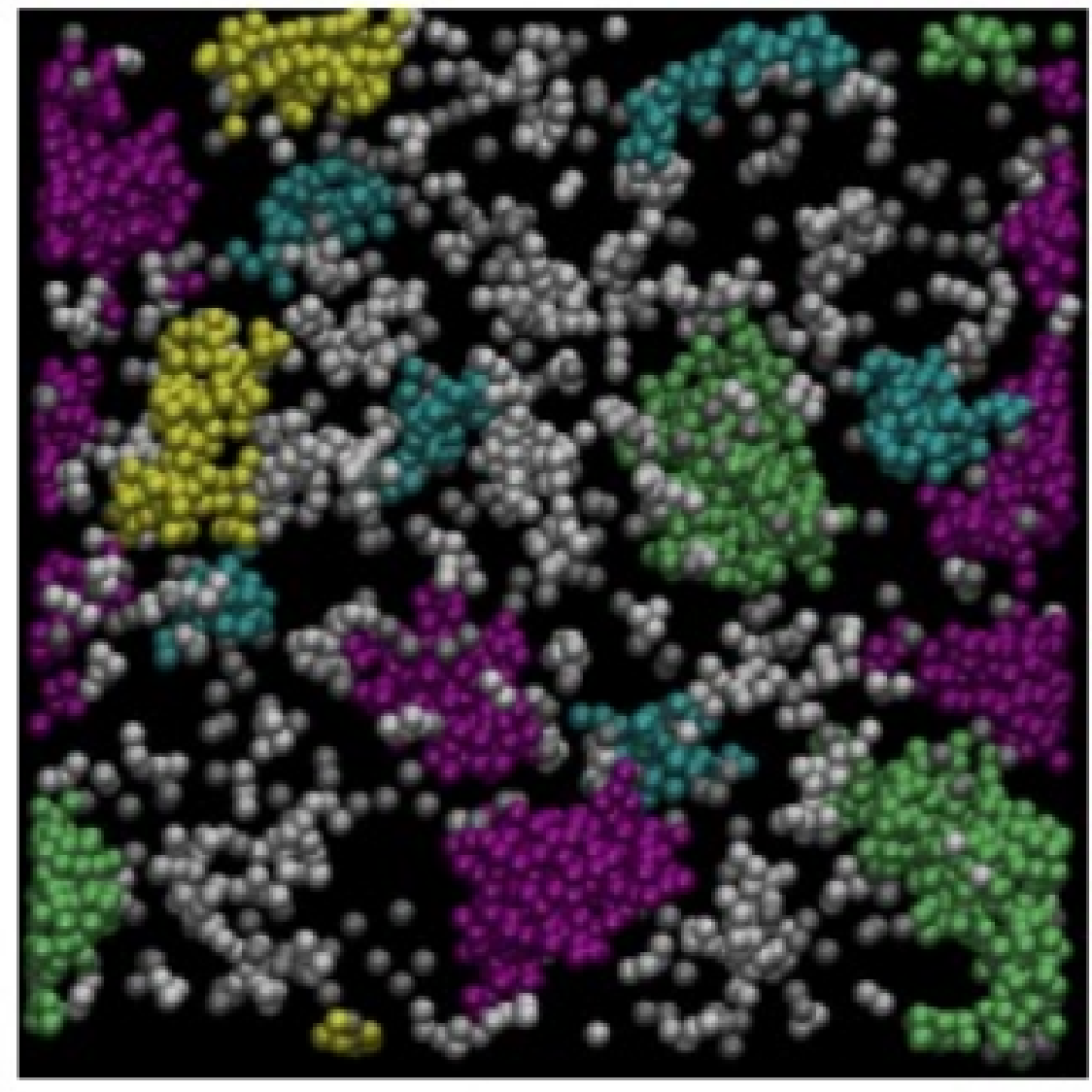

(a)

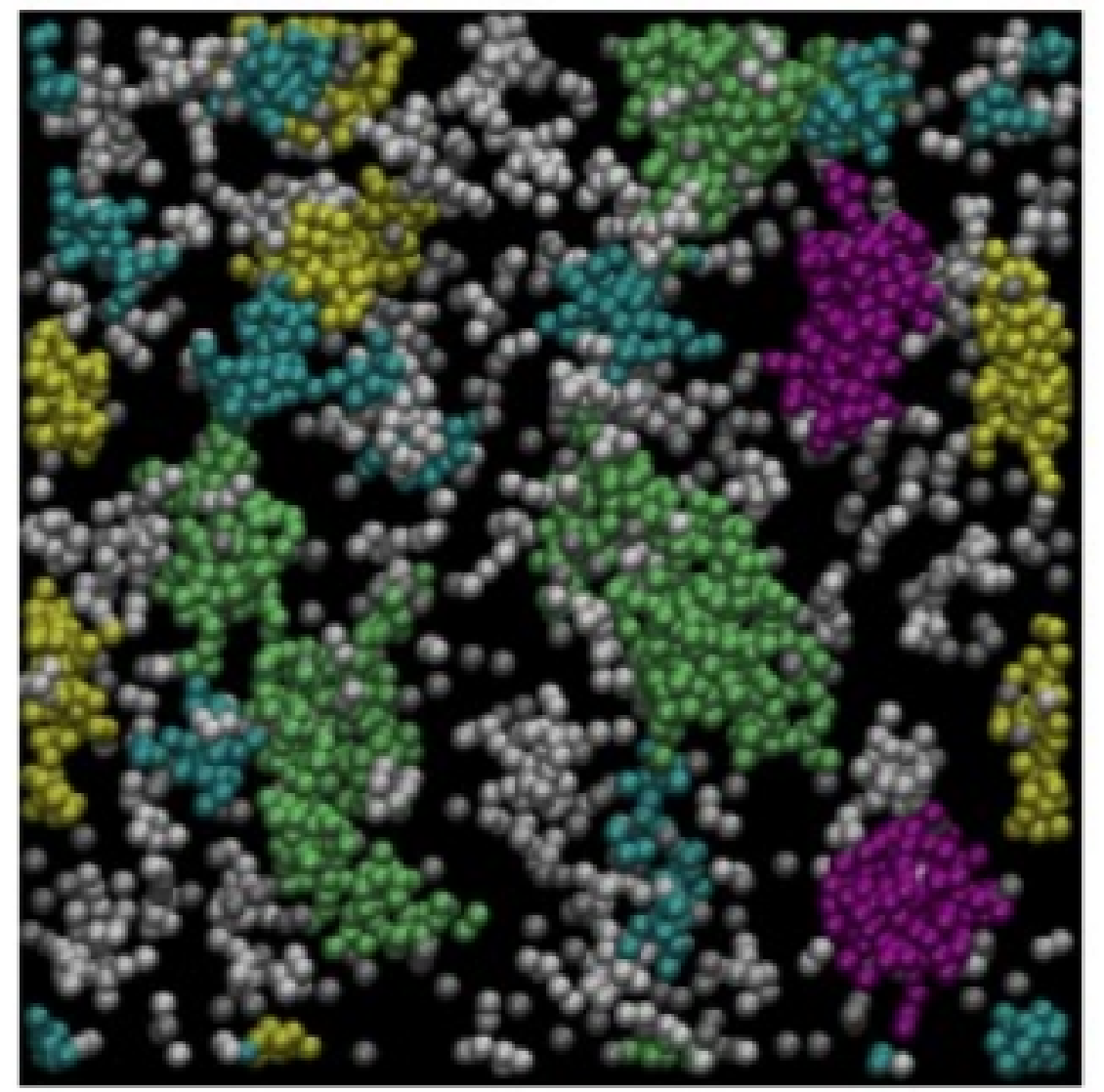

(b)

\begin{tabular}{|c|c|c|c|c|c|c|}
\hline NP color & & & & & & \\
\hline $\begin{array}{c}\text { NP size } \\
\text { (number of Mg atoms) }\end{array}$ & 1 & $2-27$ & $28-64$ & $65-125$ & $126-216$ & $217-343$ \\
\hline
\end{tabular}

Figure 2. $3 \mathrm{D}$ visualization of the $\mathrm{NPs}$ in $0.10 \mathrm{MgO}-0.90 \mathrm{SiO}_{2}$ (a) and $0.01 \mathrm{Eu}_{03 / 2}-0.10 \mathrm{MgO}-0.89 \mathrm{SiO}_{2}$ (b) glasses. Only $\mathrm{Mg}$ atoms are represented with a color code related to the size of the NP in which they are located. Two Mg atoms separated by less than $4.3 \AA$ (first minimum of the MgMg RDF) are considered to belong to the same NP. 
An $\mathrm{O}$ atom is bounded to a $\mathrm{Si}$ atom if both are separated by less than $2.1 \AA$ (i.e., the first minimum of the $\mathrm{SiO} \mathrm{RDF})$. Oxygen atom bounded to two silicon atoms is denoted bridging oxygen (BO) as it fully participates to the $\mathrm{SiO}_{2}$ network. For an ideal $\mathrm{SiO}_{2}$ glass, $100 \%$ of the $\mathrm{O}$ atoms are $\mathrm{BO}(99.3 \%$ with our model). $\mathrm{BO}$ also bounded to one $\mathrm{Mg}^{2+}$ ion (i.e., if the two atoms are separated by less than $2.9 \AA$, the first minimum of the $\mathrm{MgO}$ $\mathrm{RDF})$ is called $\mathrm{BO}^{*}$. When they are introduced in vitreous silica, $\mathrm{Mg}^{2+}$ ions create non bridging oxygen (NBO) atoms that are bounded only to one silicon atom. ${ }^{16}$ NBO bounded to one $\mathrm{Mg}$ ion is denoted $\mathrm{NBO} *$, whereas NBO bounded to two $\mathrm{Mg}^{2+}$ ions is denoted $\mathrm{NBO}^{* *}$. Finally, an oxygen atom only bounded to $\mathrm{Mg}$ ions is called NNO. Fig. 3, we show the MgO RDF and its contributions due to the previously defined oxygen types. From the different RDF curves, $\mathrm{Mg}^{2+}-\mathrm{O}$ coordinations were obtained according to the oxygen types (Tab.1).
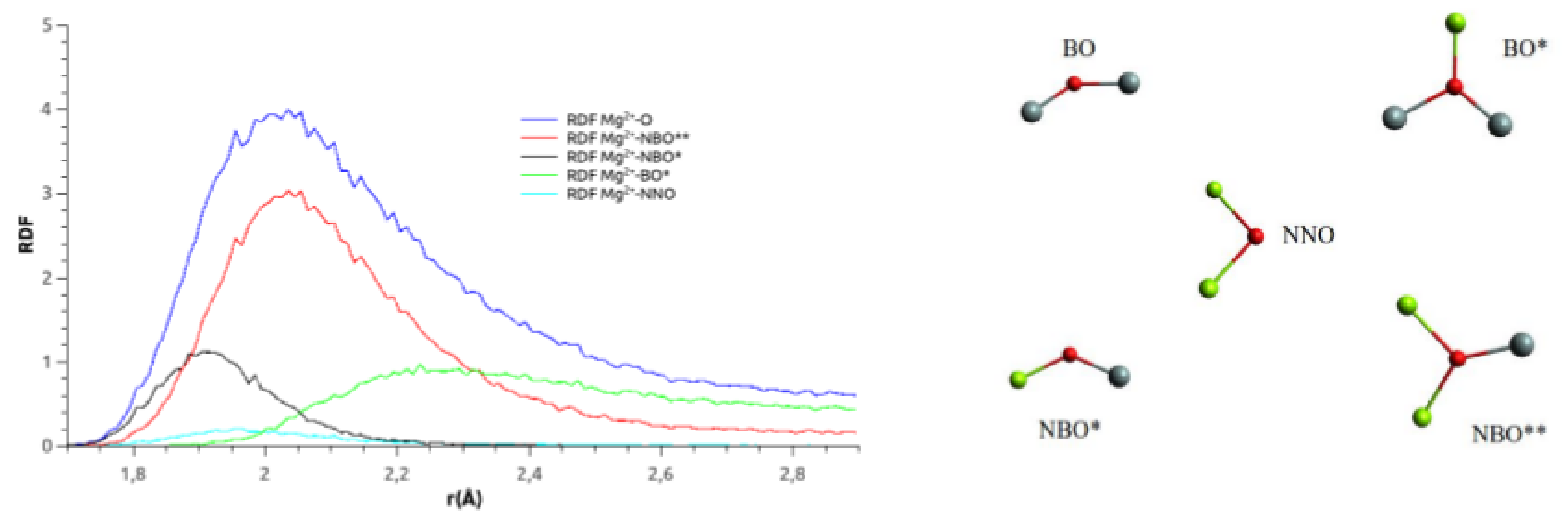

Figure 3. Different Oxygen type contributions to the RDF Mg-O, and classification of the different types of oxygen atoms (red, grey and green spheres represent oxygen, silicon and magnesium atoms respectively).

\begin{tabular}{cccccccc}
\hline $\begin{array}{c}\text { NP size } \\
\text { (number of Mg atoms) }\end{array}$ & NNO & NBO* $^{*}$ & $\mathrm{NBO}^{* *}$ & $\mathrm{BO}^{*}$ & total & $\begin{array}{c}\text { average MgO } \\
\text { composition (\%) }\end{array}$ & $\begin{array}{c}\text { fraction of Mg atoms } \\
\text { per NP size (\%) }\end{array}$ \\
\hline isolated Mg & 0.0 & 1.9 & 0.0 & 3.7 & 5.6 & 2 & 10.7 \\
$2-8$ & 0.0 & 0.9 & 2.2 & 2.7 & 5.8 & 17 & 16.9 \\
$9-125$ & 0.1 & 0.4 & 3.6 & 2.0 & 6.1 & 25 & 26.1 \\
$126-512$ & 0.2 & 0.3 & 4.2 & 1.5 & 6.2 & 32 & 46.3 \\
\hline In average & 0.1 & 0.6 & 3.2 & 2.1 & 6.0 & - & - \\
\hline
\end{tabular}

Table 1. Mg-O coordination, MgO composition and percentage of $\mathrm{Mg}$ involved by NP size. By counting Mg atoms inside a NP and $\mathrm{Si}$ atoms at a distance smaller than $4 \AA$ from a Mg (first minimum of the SiMg RDF), MgO composition of a NP is defined.

Thereafter we could analyze the local environment of a $\mathrm{Mg}^{2+}$ ion according to the size of the NP in which it is embedded (see Table 1). An isolated $\mathrm{Mg}^{2+}$ is surrounded by 5.6 oxygen atoms: $1.9 \mathrm{NBO}^{*}$ on one hand, $3.7 \mathrm{BO}^{*}$ on the other hand. There is no $\mathrm{Mg}^{2+}-\mathrm{NBO}^{* *}$ coordination since the ion is isolated in the silica matrix. When the NP size increases, $\mathrm{Mg}^{2+}-\mathrm{O}$ coordination and $\mathrm{MgO}$ concentration increase correlatively: $\mathrm{Mg}^{2+}$ ion are less and less surrounded by $\mathrm{NBO}^{*} \mathrm{~s}$ and $\mathrm{BO}^{*}$ s, and they experience a rich-NBO** environment. $\mathrm{Mg}^{2+}-\mathrm{O}$ coordination increases up to 6.2 in the largest nanoparticles. Consequently, we can observe that the larger the NPs are, the more Mg-concentrated they are, and the richer in oxygen they are.

The Fig. 4 shows EuMg RDF and EuMg cumulative distribution function (CDF). In average, $\mathrm{Eu}^{3+}$ ions are surrounded by $2.5 \mathrm{Mg}$ at $4.6 \AA$ (i.e., the first minimum of EuMg RDF). EuMg RDF presents a well defined second peak meaning that $\mathrm{Mg}$ atoms are also present in the second cationic coordination sphere. We can conclude that rare-earth ions are mainly located within the NPs, or at least in their near vicinity. The compositional analysis of the NPs (see Table 2) shows that the larger a NP, the higher its MgO content. The rare-earth fraction also increases with the NP size. Whatever the NP size, $\mathrm{Eu}^{3+}$ ions inside are over-concentrated when compared to the nominal concentration of $1 \%$. This can be explained by the oxygen-rich environment encountered in the NPs. 


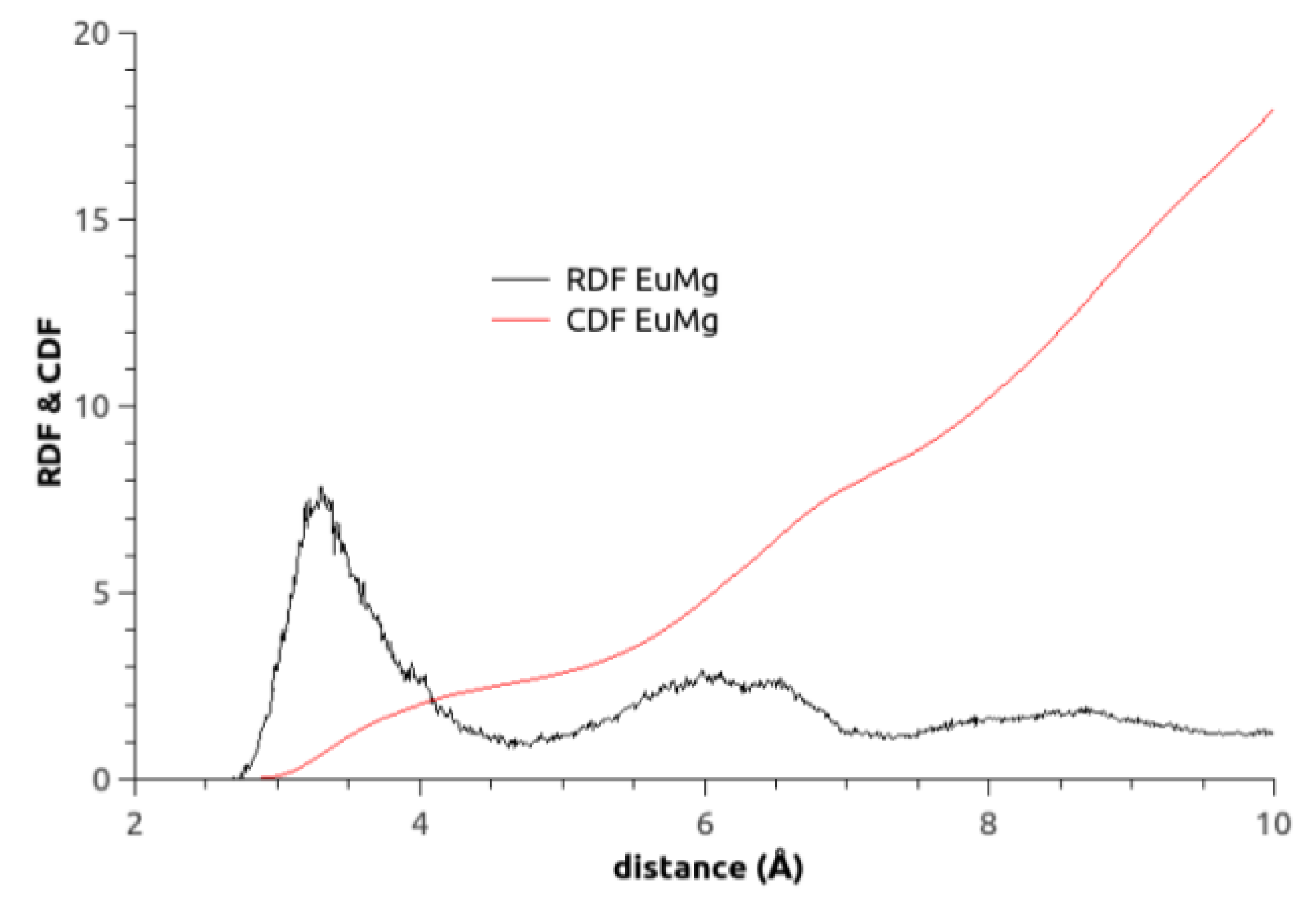

Figure 4. EuMg RDF and $\mathrm{CDF}$ in the $0.01 \mathrm{EuO}_{3 / 2}-0.10 \mathrm{MgO}-0.89 \mathrm{SiO}_{2}$.

\begin{tabular}{cccc}
\hline $\begin{array}{c}\mathrm{NP} \text { size } \\
\text { (number of Mg atoms) }\end{array}$ & $\% \mathrm{SiO}_{2}$ & $0.01 \mathrm{EuO}_{\frac{3}{2}}-0.10 \mathrm{MgO}-0.89 \mathrm{SiO}_{2}$ \\
& $\% \mathrm{MgO}$ & $\% \mathrm{EuO}_{3 / 2}$ \\
\hline $2-8$ & 82.1 & 15.7 & 2.2 \\
$9-125$ & 73.5 & 24.4 & 2.1 \\
$126-512$ & 69.6 & 27.9 & 2.5 \\
\hline
\end{tabular}

Table 2. Nanoparticles compositionas a function of their size.

Globally, $\mathrm{Eu}^{3+}-\mathrm{O}$ coordination goes from 6.1 into vitreous silica doped with the same RE concentration, to 6.7 in presence of $\mathrm{Mg}$ co-doping. In average, they are surrounded by $1.6 \mathrm{BO}^{*}, 1.4 \mathrm{NBO}^{*}, 3.5 \mathrm{NBO}^{* *}$ and $0.2 \mathrm{NNO}$. These values confirm that the rare-earth ions are embedded in the NBO-rich environment provided by the NP.

\subsection{Rare-earth clustering}

We have also considered the effects of $\mathrm{Mg}$ co-doping on the rare-earth ions clustering (see Table 3 ). $\mathrm{RE}$ ions are categorized according to the size of the cluster they form: isolated RE ions are called $1 \mathrm{R}$. RE ions forming a pair are denoted $2 \mathrm{R}$, and $3 \mathrm{R}+$ indicates $\mathrm{RE}$ ions within clusters composed of three or more RE ions. In average, $73 \%$ of $\mathrm{Eu}^{3+}$ are isolated, this fraction goes down to $67 \%$ in a silica matrix without Mg-codoping. Globally, it seems that Mg co-doping tends to limit the RE clustering. Within NPs, RE ions are over-concentrated: for example, the $\mathrm{Eu}^{3+}$ ions concentration is $2.5 \%$ into the largest NPs, wherein they are $69 \%$ isolated and $20 \% 3 \mathrm{R}+$. Without $\mathrm{Mg}$ co-doping, in silica doped also with $2.5 \%$ of $\mathrm{Eu}^{3+}, 27 \%$ are $3 \mathrm{R}+$ and only $48 \%$ remain isolated. Therefore, we can conclude that the RE ions are mainly found in the NPs and that the clustering is however limited. This can be explained by the oxygen-rich environment together with a structuring of this environment by Mg ions. 


\begin{tabular}{cccccc}
\hline System & $\begin{array}{c}\text { NP size } \\
\text { (In the silica matrix) }\end{array}$ & RE & $1 \mathrm{R}$ & $2 \mathrm{R}$ & $3 \mathrm{R}+$ \\
\hline \hline & 0 (isolated RE) & 12.7 & 97 & 3 & 0 \\
$0.01 \mathrm{Eu0}_{3 / 2}-0.10 \mathrm{MgO}-0.89 \mathrm{Si0}_{2}$ & 1 & 33.0 & 58 & 20 & 22 \\
& $2-8$ & 28.8 & 69 & 14 & 17 \\
& $9-125$ & 29.4 & 58 & 29 & 13 \\
& $126-512$ & 31.0 & 69 & 11 & 20 \\
& average & - & $\mathbf{7 3}$ & $\mathbf{1 5}$ & $\mathbf{1 2}$ \\
\hline $0.01 \mathrm{Eu0}_{3 / 2}-0.99 \mathrm{SiO}_{2}$ & & - & $\mathbf{6 7}$ & $\mathbf{2 1}$ & $\mathbf{1 2}$ \\
\hline $0.025 \mathrm{EuO}_{3 / 2}-0.97 .5 \mathrm{SiO}_{2}$ & & - & 48 & 25 & 27 \\
\hline
\end{tabular}

Table 3. Europium clustering

\subsection{Influence of uniaxial stress}

Fig. 5 shows the distribution of NP size before and after elongation (i.e., $\epsilon=0$ and $\epsilon=8$ ) for the three drawing temperatures (i.e., $1900 \mathrm{~K}, 2150 \mathrm{~K}$ and $2300 \mathrm{~K}$ ) at the same strain rate $\nu=10^{9} \mathrm{~s}^{-1}$. For $\epsilon=8$, the red distribution corresponds to the case of the preform not deformed but heated at the drawing temperature, for the same duration. Comparing red and blue distributions allows to get insight about thermodynamical and rheological contributions on the modification of the $\mathrm{Mg}$ atoms distribution during the drawing step. All along the plastic deformation, the uniaxial stress remains almost constant at $3 \mathrm{GPa}, 2.1 \mathrm{GPa}$ and $1.5 \mathrm{GPa}$ for $\mathrm{T}=1900 \mathrm{~K}, \mathrm{~T}=$ $2150 \mathrm{~K}$ and $\mathrm{T}=2300 \mathrm{~K}$, respectively. At the highest temperature, we can see that the blue distribution is almost covered by the red one, meaning that the stress has a minor effect on the NP size compared to thermodynamical processes. At lowest temperature, viscous stress becomes the major contribution and it leads to elongation and break up of the nanoparticles. In the light of these findings, the following trend can be proposed: the lower the drawing temperature is, the higher the viscous stress is, the smaller the nanoparticles into the fiber become.
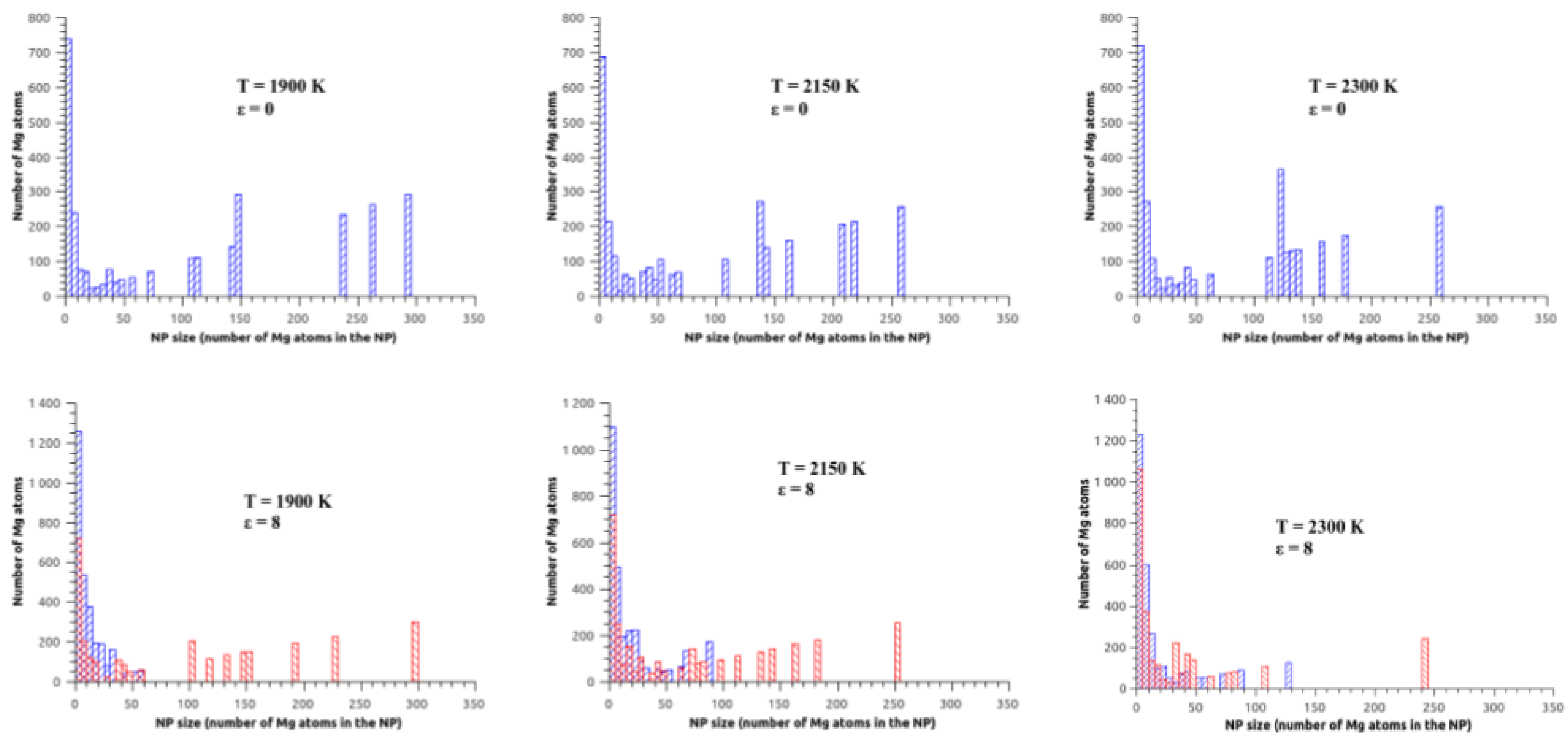

Figure 5. Drawing effect on the NP size distribution for different drawing temperatures. 


\section{CONCLUSION}

We have investigated the environment encountered by rare-earth ions into vitreous silica co-doped with magnesium. By using a potential designed to reproduce phase separation into silicate glass, we obtained Mg-silicate nanoparticles embedded in silica matrix. Rare-earth ions have been mainly localized into nanoparticles where they are over-concentrated against the nominal doping concentration. Despite of over-concentration, the clustering of rare-earth ions is significantly limited because of the environment encountered in the Mg-rich NPs. The nanostructured preform was then drawn to a fiber at different temperatures and a viscous stress effect on the nanoparticles size distribution was noticed. It appeared that the nanoparticles became smaller with the decrease of the drawing temperature. Works are in progress to study the effect of strain rate variations.

\section{ACKNOWLEDGMENTS}

This study was funded by Université d'Angers and Agence Nationale de la Recherche (ANR-14-CE07-0016-01, Nice-DREAM).

\section{REFERENCES}

[1] Blanc, W. et al., "Fabrication of rare earth-doped transparent glass ceramic optical fibers by modified chemical vapor deposition," J. Non-Cryst. Solids 401, 50-53 (2011).

[2] D'Acapito, F., Blanc, W., and Dussardier, B., "Different er3+ environments in mg-based nanoparticle-doped optical fibre preforms," J. Non-Cryst. Solids 401, 50-53 (2014).

[3] Lochhead, V. J. and Bray, K. L., "Rare-Earth Clustering and Aluminium Codoping in Sol-Gel Silica: Inverstigation Using Europium(III) Fluorescence Spectroscopy," Chem. Mater. 7, 572-577 (1995).

[4] Vermillac, M. et al., "Fiber-draw-induced elongation and break-up of particles inside the of a silica-based optical fiber.," J. Amer. Ceram. Soc. 100(5), 1814-1819 (2017).

[5] X.Bidault, Chaussedent, S., and Blanc, W., "A simple transferable adaptive potential to study phase separation in large-scale xmgo-(1-x)sio2 binary glasses," J. Chem. Phys. 143, 154501 (2015).

[6] Hageman, V. B. M. and Oonk, H. A. J., "Liquid immiscibility in the $\mathrm{SiO} 2+\mathrm{MgO}, \mathrm{SiO} 2+\mathrm{SnO}, \mathrm{SiO} 2+\mathrm{La} 2 \mathrm{O} 3$, and $\mathrm{SiO} 2+\mathrm{Y} 2 \mathrm{O} 3$ systems," Phys. Chem. Glas. 27, 194-198 (1986).

[7] Plimpton, S. J. Comput. Phys. 117, 1 (1995).

[8] Pedone, A., Malavasi, G., Menziani, M., Cormack, A., and U.Segre, "A new self-consistent empirical interatomic potential model for oxides, silicates, and silica-based glasses," J. Phys. Chem. B 110, 11780 (2006).

[9] Afify, N., Mountjoy, G., and Haworth, R., "Selecting reliable interatomic potentials for classical molecular dynamics simulations of glasses: The case of amorphous sio2," Computational Materials Science 128, 75-80 (2017).

[10] Wolf, D., Keblinski, P., Phillpot, S. R., and Eggebrecht, J. J. Chem. Phys. 110, 8254 (1999).

[11] Hudon, P. and Baker, D. R., "The nature of phase separation in binary oxide melts and glasses. i. silicate systems," J. Non-Cryst. Solids 303, 299-345 (2002).

[12] Bidault, X., Chaussedent, S., Blanc, W., and Neuville, D. R., "Deformation of silica glass studied by molecular dynamics: Structural origin of the anisotropy and non-newtonian behavior," J. Non-Cryst. Solids 433, 38-44 (2016).

[13] Humphrey, W., Dalke, A., and Schulten, K., "VMD - Visual Molecular Dynamics," Journal of Molecular Graphics 14, 33-38 (1996).

[14] Blanc, W., Guillermier, C., and Dussardier, B., "Composition of nanoparticles in optical fibers by secondary ion mass spectrometry," Opt. Mater. Express 2, 1504 (2012).

[15] Francois-Saint-Cyr, H. et al., "Correlative compositional analysis of fiber-optic nanoparticles," Microsc. Microanal. 20, 994-995 (2014).

[16] Al-Hasni, B. M. and Mountjoy, G., "A molecular dynamics study of the atomic structure of $\mathrm{x}(\mathrm{MgO}) 100-$ x(SiO2)," J. Non-Cryst. Solids 389, 33-44 (2014). 